intracoronary $\mathrm{p}$-Selectin and activated PMA $\left[\mathrm{r}^{2}=0.5 ; \mathrm{p}=0.03, \mathrm{r}^{2}=0.9\right.$; $\mathrm{p}=0.0008$ respectively]. PMP correlated positively with intracoronary $\mathrm{p}$-selectin and activated PMA $\left[\mathrm{r}^{2}=0.7 ; \mathrm{p}=0.01\right.$ and $\mathrm{r}^{2}=0.6$; $\mathrm{p}=0.02$ respectively] but they also correlated with $\mathrm{TF}+\mathrm{PMA}$ $\left[\mathrm{r}^{2}=0.7 ; \mathrm{p}=0.01\right]$. CD62P + PMP was strongly positively related with intracoronary $\mathrm{p}$-Selectin $\left[\mathrm{r}^{2}=0.9 ; \mathrm{p}=0.0001\right]$. CD105+ EMP also correlated positively with intracoronary p-selectin, activated $\mathrm{PMA}$ and TF+ PMA $\left[\mathrm{r}^{2}=0.7 ; \mathrm{p}=0.007, \mathrm{r}^{2}=0.6 ; \mathrm{p}=0.01\right.$ and $\mathrm{r}^{2}=0.5$; $\mathrm{p}=0.04$ for intracoronary $\mathrm{p}$-selectin, activated PMA and TF+ PMA respectively]. $\mathrm{CD} 62 \mathrm{E}+\mathrm{EMP}$ positively correlated with intracoronary p-selectin, activated PMA and TF+ PMA $\left[\mathrm{r}^{2}=0.6 ; \mathrm{p}=0.02\right.$, $\mathrm{r}^{2}=0.6 ; \mathrm{p}=0.01$ and $\mathrm{r}^{2}=0.5 ; \mathrm{p}=0.03$ respectively].

Conclusions Markers of platelet activation at the site of the culprit lesion during ST elevation ACS correlated strongly not only with total MPs and PMPs but also with endothelial derived MPs. The interaction between activated platelets and monocytes with endothelial cells and the subsequent formation of PMP and EMP during ACS highlights the importance of the downstream effect of activated platelets, monocytes and endothelial cells via MP formation and their contribution in the pathophysiology of ACS.

\section{IMPLICATIONS OF LOWERING THE THRESHOLD OF CARDIAC TROPONIN IN THE DIAGNOSIS OF MYOCARDIAL INFARCTION}

doi:10.1136/heartjnl-2012-301877b.128

${ }^{1} \mathrm{~K}$ K Lee, ${ }^{*}{ }^{1} \mathrm{~N} L$ Mills, ${ }^{2} \mathrm{D}$ A McAllister, ${ }^{1} \mathrm{~A} M \mathrm{D}$ Churchhouse, ${ }^{3} \mathrm{M}$ MacLeod, ${ }^{4} \mathrm{M}$ Stoddart, ${ }^{4} \mathrm{~S}$ Walker, ${ }^{1} \mathrm{M}$ A Denvir, ${ }^{1} \mathrm{~K}$ A A Fox, ${ }^{1} \mathrm{D}$ E Newby. ${ }^{1}$ Centre for Cardiovascular Science, Edinburgh University, Edinburgh, UK; ${ }^{2}$ Public Health Sciences, Edinburgh University, Edinburgh, UK; ${ }^{3}$ Edinburgh Heart Centre, Royal Infirmary of Edinburgh, Edinburgh, UK; ${ }^{4}$ Department of Clinical Biochemistry, Royal Infirmary of Edinburgh, Edinburgh, UK

Introduction The Universal Definition recommends the 99 percentile of cardiac troponin as the diagnostic threshold for myocardial infarction (MI) in patients with suspected acute coronary syndrome if the assay achieves a coefficient of variation (CV) $<10 \%$. However, due to imprecision in contemporary assays and concern of over diagnosing myocardial infarction, diagnostic thresholds are currently set at higher concentrations where the assay can achieve $\mathrm{CV} \leq 10 \%$. The aim of this study was to assess the relationship between plasma troponin I concentrations, assay precision and clinical outcomes in patients with suspected acute coronary syndrome.

Methods Using a contemporary sensitive troponin I assay, consecutive patients admitted with suspected acute coronary syndrome $(\mathrm{n}=2092)$ were stratified according to the 99 th percentile $(0.012 \mathrm{ng} /$ $\mathrm{ml}$; CV 20.8\%) and current diagnostic threshold $(0.05 \mathrm{ng} / \mathrm{ml}$; CV $7.2 \%$ ) into three groups: $<0.012 \mathrm{ng} / \mathrm{ml}, 0.012-0.049 \mathrm{ng} / \mathrm{ml}$ and $\geq 0.05 \mathrm{ng} / \mathrm{ml}$. Event-free survival (recurrent myocardial infarction or death) at 1 year was compared between patients grouped by troponin I concentration.

Results Plasma troponin concentrations were $<0.012 \mathrm{ng} / \mathrm{ml}$ in 988 patients (47\%), $0.012-0.049 \mathrm{ng} / \mathrm{ml}$ in 352 patients $(17 \%)$ and $\geq 0.05 \mathrm{ng} / \mathrm{ml}$ in 752 patients $(36 \%)$. At 1 year, patients with troponin concentrations $0.012-0.049 \mathrm{ng} / \mathrm{ml}$ were more likely to be dead or readmitted with recurrent myocardial infarction compared to those with troponin concentrations $<0.012 \mathrm{ng} / \mathrm{ml}(13 \%$ vs $3 \%$; OR 4.8, 95\% CI 3.0 to $7.7 ; \mathrm{p}<0.001)$. Compared to troponin $\geq 0.050 \mathrm{ng} / \mathrm{ml}$, patients with troponin $0.012-0.049 \mathrm{ng} / \mathrm{ml}$ had a higher risk profile but were less likely to be diagnosed with, or investigated and treated for, acute coronary syndrome.

Conclusions Lowering the diagnostic threshold to the 99th percentile and accepting greater assay imprecision would identify those at high-risk of recurrent MI and death, but increase the diagnosis of MI by $46 \%$. It remains to be established whether reclassifying and treating these patients as MI would improve outcome.

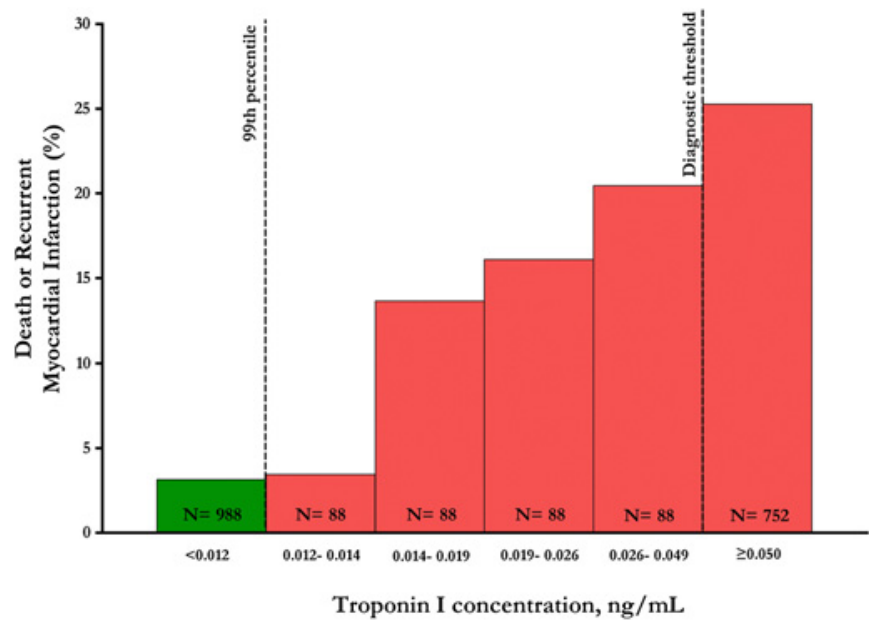

Abstract 128 Figure 1

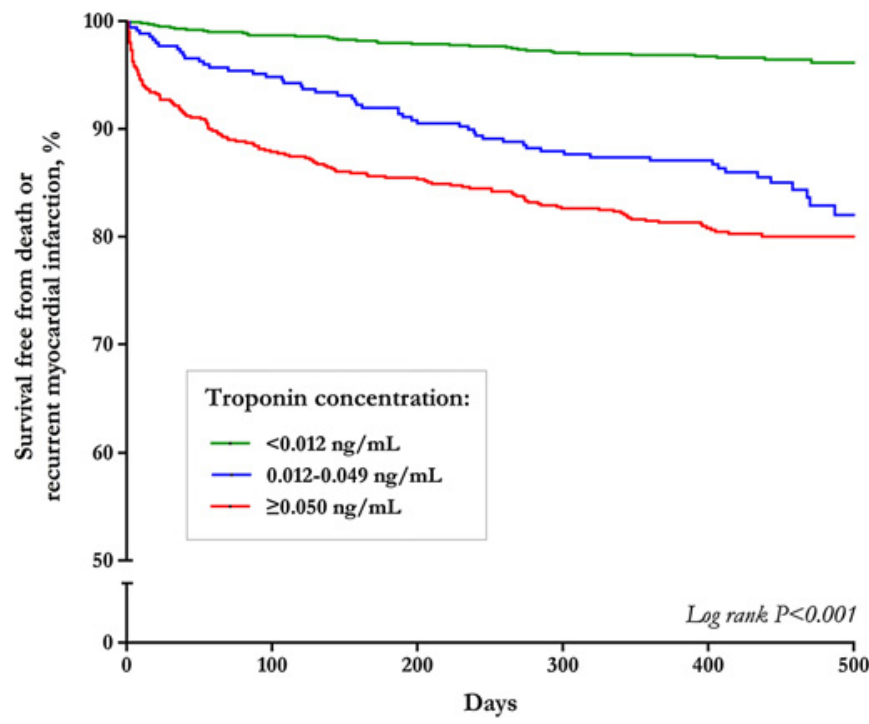

Abstract 128 Figure 2

\section{THE PROGNOSTIC; VALUE OF A 7-WEEK HIGH SENSITIVITY TROPONIN T LEVEL AFTER AN ACUTE CORONARY SYNDROME}

doi:10.1136/heartjnl-2012-301877b.129

D S Ang, * M P Kao, C C Lang, E Dow, A D Struthers. University of Dundee, Dundee, UK

Background High sensitivity Troponin T (HS-TnT) is a well established diagnostic and prognostic tool in acute coronary syndrome (ACS). However, its role in the convalescence phase after an ACS is unknown. Our first aim was to assess the prognostic utility of a single HS-TnT level at 7-week post ACS. Second, we evaluated whether any serial changes in HS-TnT between the index admission and 7 weeks post ACS had any link with prognosis. Third, we assessed whether the prognostic utility of HS-TnT is independent of various echocardiographic abnormalities.

Methods We measured HS-TnT levels in 326 consecutive patients at 7 weeks after an ACS event. The composite end point of death from any cause or acute myocardial infarction (AMI) was evaluated over a median duration of 30 months. 
Results Patients in the third HS-TnT tercile were 6 times more likely to sustain a cardiovascular event compared to those in the first tercile [unadjusted RR 6.11 (95\% CI 2.98 to 12.50)]. A high HS-TnT predicted adverse clinical outcomes independent of conventional risk factors, left ventricular dysfunction and left ventricular hypertrophy on echocardiography [adjusted RR 2.69 (95\% CI 1.45 to 5.00)]. Patients with persistent HS-TnT elevation at 7 weeks were also at an increased risk of cardiovascular events compared to those with an initial high HS-TnT which then normalised [unadjusted RR 3.39 (95\% CI 2.02 to 5.68$)$ ].

Conclusion We have demonstrated for the first time the prognostic utility of a single 7-week HS-TnT measurement in routine ACS patients and that it could be used to assist medium term risk stratification in this patient cohort. Future studies should evaluate whether tailoring specific treatment interventions to higher risk individuals as identified by an elevated HS-TnT during the convalescence phase of ACS would improve clinical outcomes.

\section{PRIOR CORONARY ARTERY BYPASS GRAFT PATIENTS TREATED WITH PRIMARY PERCUTANEOUS CORONARY INTERVENTION HAVE HIGHER LONG-TERM ADVERSE EVENT RATES}

doi:10.1136/heartjnl-2012-301877b.130

S M Hamshere,* D A Jones, M Akhtar, K S Rathod, A Graham, 0 Guttmann, S Gallagher, A Jain, C Knight, A Mathur, A Wragg. London Chest Hospital, London, UK

Background Limited information exists regarding procedural success and clinical outcomes of ST-segment elevation myocardial infarction (STEMI) patients with previous CABG undergoing primary PCI. We sought to compare outcomes in STEMI patients undergoing primary percutaneous coronary intervention (PCI) with or without previous coronary artery bypass grafts (CABG).

Methods Clinical information was analysed from a prospective database on 2322 STEMI patients who underwent Primary PCI between January 2004 and May 2010 at a London centre. 104 of 2322 (4.5\%) patients had prior CABG. MACE was defined as a composite of death, stroke and target vessel revascularisation (TVR). Outcome assessed by all-cause mortality information provided by the Office of National Statistics via the BCIS CCAD national audit.

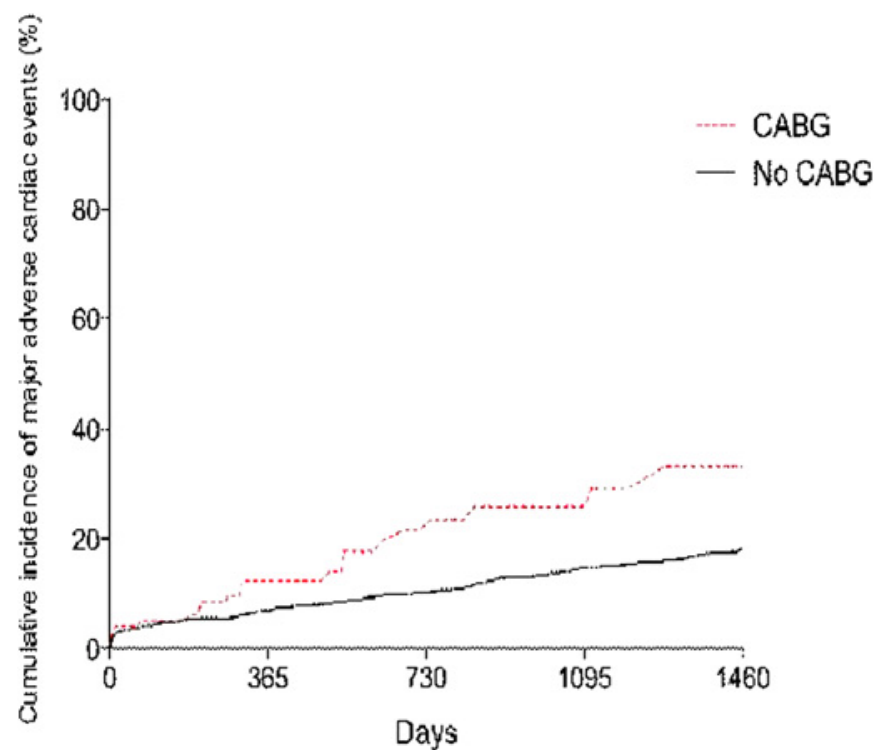

Abstract 130 Figure 1
Results Patients with previous CABG were older, had more associated comorbidity and a higher incidence of multivessel coronary disease than patients who have never had CABG. In patients with previous $C A B G$, the infarct related artery (IRA) was split evenly between a bypass graft $(n=50)$ and a native vessel $(n=54)$. Procedural success (defined as TIMI 3 flow at the end of procedure) was less likely in patients with previous CABG $84.5 \%$ vs $95.6 \%$ $(p<0.0001)$ than in patients who had never undergone CABG. Patients with previous CABG had more MACE $(32.8 \%$ vs $16.5 \%$, $\mathrm{p}=0.01$ ) during the 4-year follow-up period (Abstract 130 figure 1). After multivariable adjustment this difference persisted (HR 2.2, $95 \%$ CI 1.26 to $3.78, p=0.02$ ). When stratifying prior CABG patients by the type of IRA (Abstract 130 figure 2); Long term MACE were significant more likely in patients who had bypass graft PCI than in patients that had native vessel PCI (44.6\% vs $19.8 \%, p=0.04)$.

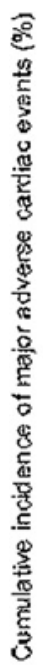

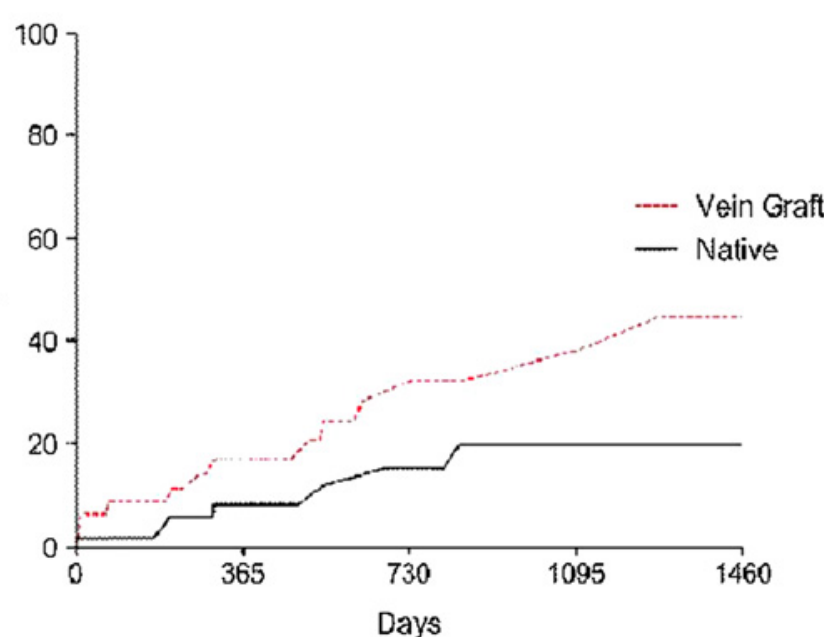

Abstract 130 Figure 2

Conclusions Previous CABG patients with STEMI treated with primary PCI have higher long-term adverse events. The long-term event rates are higher still if the IRA is a bypass graft.

\section{MULTIVESSEL CORONARY DISEASE IN PRIMARY PERCUTANEOUS INTERVENTION. REVASCULARISATION STRATEGY AND ITS IMPACT ON MEDIUM TERM MORTALITY}

doi:10.1136/heartjnl-2012-301877b.131

A E Alahmar,* A S Banning, N S Rajendra, B Wrigley, K Pujara, A H Gershlick. Glenfield Hospital, University Hospitals of Leicester NHS Trust, Leicester, UK

Introduction The optimal revascularisation strategy for patients presenting with ST-Segment Elevation MI (STEMI) and multivessel coronary disease remains under debate. Although current practice in patients without cardiogenic shock is to treat the infarct-related artery only at the time of presentation, it is not clear whether residual coronary disease should be treated either during the acute admission or post-discharge.

Methods All Patients presenting to UHL between April 2008 and November 2011 with STEMI undergoing Primary PCI were analysed retrospectively. The outcome measure was mortality either inhospital or post-discharge. All patients were followed-up until end of November 2011, follow-up duration is expressed as mean \pm SD. Patients were divided into three groups; PCI for single vessel disease (SVD), Multivessel disease (MVD) \& single vessel PCI and MVD \& multivessel PCI. Baseline characteristics (age, gender, risk factors, 